Homology, Homotopy and Applications, vol.16(1), 2014, pp.167-177

\title{
KEI MODULES AND UNORIENTED LINK INVARIANTS
}

\author{
MICHAEL GRIER AND SAM NELSON
}

(communicated by Ronald Brown)

\begin{abstract}
We define invariants of unoriented knots and links by enhancing the integral kei counting invariant $\Phi_{X}^{\mathbb{Z}}(K)$ for a finite kei $X$ using representations of the kei algebra, $\mathbb{Z}_{K}[X]$, a quotient of the quandle algebra $\mathbb{Z}[X]$ defined by Andruskiewitsch and Graña. We give an example that demonstrates that the enhanced invariant is stronger than the unenhanced kei counting invariant. As an application, we use a quandle module over the Takasaki kei on $\mathbb{Z}_{3}$ which is not a $\mathbb{Z}_{K}[X]$-module to detect the noninvertibility of a virtual knot.
\end{abstract}

\section{Introduction}

In [10], Mituhisa Takaski introduced an algebraic structure known as kei (or 圭 in the original kanji). In [7] this same structure was reintroduced under the name involutory quandle, a special case of a more general algebraic structure related to oriented knots known as quandles. These algebraic structures can be understood as arising from the unoriented and oriented Reidemeister moves respectively via a certain labeling scheme, encoding knot structures in algebra.

In $[\mathbf{1}]$, an associative algebra $\mathbb{Z}[X]$ was defined for every finite quandle $X$; in $[\mathbf{3}]$ a geometric interpretation of $\mathbb{Z}[X]$ was given, with generators representing coefficients of "beads" indexed by quandle labelings of arcs, with relations defined from the Reidemeister moves. Representations of $\mathbb{Z}[X]$, known as quandle modules, were used to define new invariants of oriented knots and links in $[\mathbf{3}]$. In $[\mathbf{8}]$ a modification of $\mathbb{Z}[X]$ for finite racks (a generalization of quandles to the case of blackboard-framed isotopy) was used to define invariants of framed and unframed oriented knots and links.

In this paper we define a modification of the quandle algebra we call the kei algebra $\mathbb{Z}_{K}[X]$ and use it to extend the invariants defined in $[8]$ to unoriented knots and links. The paper is organized as follows. In section 2 we review the basics of kei and the kei counting invariant. In section 3 we define the kei algebra and kei modules. In section 4 we define the kei module enhanced counting invariant. As an application, we use a module over $\mathbb{Z}[X]$ for a kei $X$ which is not a $\mathbb{Z}_{K}[X]$-module to detect the

Received August 24, 2011, revised May 24, 2013; published on April 28, 2014.

2010 Mathematics Subject Classification: 57M27, 57M25.

Key words and phrases: Kei algebra, kei module, involutory quandle, enhancement of counting invariants.

Article available at http://dx.doi.org/10.4310/HHA.2014.v16.n1.a10

Copyright (C) 2014, International Press. Permission to copy for private use granted. 
non-invertibility of a virtual knot. In section 5 we collect a few questions for future research.

\section{Kei}

Kei or involutory quandles were introduced by Mituhisa Takasaki in 1945 [10] and later reintroduced independently by David Joyce and S.V. Matveev in the early 1980s $[7,9]$.

Definition 2.1. A kei or involutory quandle is a set $X$ with a binary operation $\triangleright$ satisfying for all $x, y, z \in X$

(i) $x \triangleright x=x$,

(ii) $(x \triangleright y) \triangleright y=x$, and

(iii) $(x \triangleright y) \triangleright z=(x \triangleright z) \triangleright(y \triangleright z)$.

Example 2.2. Let $X$ be any abelian group regarded as a $\mathbb{Z}$-module. Then $X$ is a kei under the operation

$$
x \triangleright y=2 y-x .
$$

Such a kei is known as a Takasaki kei. If $X \cong \mathbb{Z}_{n}$ then $X$ is often denoted as $R_{n}$ in the knot theory literature, known as the dihedral quandle on $n$ elements. $R_{n}$ can also be understood as the set of reflections of a regular $n$-gon.

Example 2.3. Let $X$ be any module over $\mathbb{Z}[t] /\left(t^{2}-1\right)$. Then $X$ is a kei known as an Alexander kei under the operation

$$
x \triangleright y=t x+(1-t) y .
$$

A Takasaki kei is an Alexander kei with $t=-1$.

Example 2.4. Let $L$ be an unoriented link diagram and let $A=\left\{a_{1}, \ldots, a_{n}\right\}$ be a set of generators corresponding bijectively with the set of arcs of $L$. The Fundamental $K e i$ of $L, F K(L)$, is defined in the following way. First, let $W(L)$ be the set of kei words in $A$, defined recursively by the rules

- $a \in A \Rightarrow a \in W(L)$ and

- $x, y \in W(L) \Rightarrow x \triangleright y \in W(L)$.

Then the free kei on $A$ is the set of equivalence classes of kei words in $A$ under the equivalence relation generated by relations of the forms

- $x \triangleright x \sim x$,

- $(x \triangleright y) \triangleright y \sim x$, and

- $(x \triangleright y) \triangleright z \sim(x \triangleright z) \triangleright(y \triangleright z)$

for all $x, y, z \in W(L)$. The free kei is a kei under the operation $[x] \triangleright[y]=[x \triangleright y]$. Now, at each crossing in $L$, we have a crossing relation given by $z=x \triangleright y$ where $y$ is the 
overcrossing arc and $x$ and $z$ are the undercrossing arcs. That is, we have

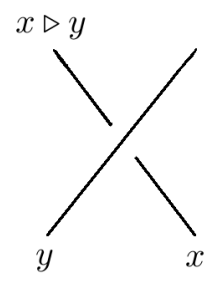

Then the fundamental kei of $L, F K(L)$, is the set of equivalence classes of free kei elements modulo the crossing relations of $L$, or equivalently $F K(L)$ is the set of equivalence classes of kei words in $A$ modulo the equivalence relation determined by the crossing relations together with the free kei relations.

It is convenient to describe a finite kei $X=\left\{x_{1}, \ldots, x_{n}\right\}$ with a matrix encoding the operation table of $X$, i.e. a matrix $M_{X}$ whose $(i, j)$ entry is $k$ where $x_{k}=x_{i} \triangleright x_{j}$. For example, the Takasaki kei on $\mathbb{Z}_{3}$ has matrix

$$
M_{X}=\left[\begin{array}{lll}
1 & 3 & 2 \\
3 & 2 & 1 \\
2 & 1 & 3
\end{array}\right]
$$

where we set $x_{1}=1, x_{2}=2$, and $x_{3}=3=0$.

As with groups and other algebraic structures, we have the following standard notions:

Definition 2.5. Let $X$ and $Y$ be kei.

- A map $f: X \rightarrow Y$ is a kei homomorphism if for all $x, x^{\prime} \in X$ we have $f\left(x \triangleright x^{\prime}\right)=$ $f(x) \triangleright f\left(x^{\prime}\right)$.

- A subset $Y \subset X$ which is itself a kei under the kei operation $\triangleright$ of $X$ is a subkei of $X$. It is easy to check that $Y \subset X$ is a subkei if and only if $Y$ is closed under $\triangleright$.

For defining invariants of unoriented links, we have the following well-known result:

Theorem 2.6. If $L$ and $L^{\prime}$ are ambient isotopic unoriented links, then there is an isomorphism of kei $\phi: F K(L) \rightarrow F K\left(L^{\prime}\right)$. For any finite kei $X$, the sets of homomorphisms $\operatorname{Hom}(F K(L), X)$ and $\operatorname{Hom}\left(F K\left(L^{\prime}\right), X\right)$ are finite and there is an induced bijection $\phi_{*}: \operatorname{Hom}(F K(L), X) \rightarrow \operatorname{Hom}\left(F K\left(L^{\prime}\right), X\right)$. In particular, the cardinality of the set of rack homomorphisms $\Phi_{X}^{\mathbb{Z}}(L)=|\operatorname{Hom}(F K(L), X)|$ is a non-negative integervalued invariant of unoriented links known as the integral kei counting invariant.

A kei homomorphism $f: F K(L) \rightarrow X$ can be represented as a labeling of the $\operatorname{arcs}$ of $L$ with elements of $X$ satisfying the crossing relations at every crossing - such a labeling defines a unique homomorphism, and every $f \in \operatorname{Hom}(F K(L), X)$ can be so represented.

Example 2.7. We can use the kei counting invariant to see that the trefoil knot $3_{1}$ is nontrivially knotted. Let $X$ be the Takasaki kei on $\mathbb{Z}_{3}$; we have $x \triangleright y=2 y-x=$ 
$2 y+2 x$. The crossing relations in $3_{1}$ give us the system of linear equations

$$
\begin{aligned}
& z=2 x+2 y \\
& y=2 z+2 x \\
& x=2 x+2 y
\end{aligned} \rightarrow\left[\begin{array}{lll}
2 & 2 & 2 \\
2 & 2 & 2 \\
2 & 2 & 2
\end{array}\right] \rightarrow\left[\begin{array}{lll}
1 & 1 & 1 \\
0 & 0 & 0 \\
0 & 0 & 0
\end{array}\right]
$$

and the solution space is two-dimensional, giving us a total of $\Phi_{X}^{\mathbb{Z}}\left(3_{1}\right)=9$ solutions. Since $\Phi_{X}^{\mathbb{Z}}($ Unknot $)=3$, the integral kei counting invariant detects the knottedness of the trefoil.

Remark 2.8. Replacing the second kei axiom with the alternative axiom

$\left(\right.$ ii $\left.^{\prime}\right)$ There exists a second operation $\triangleright^{-1}$ satisfying

$$
(x \triangleright y) \triangleright^{-1} y=x=\left(x \triangleright^{-1} y\right) \triangleright y
$$

for all $x, y \in X$

yields an algebraic object known as a quandle, which is the oriented analog of kei. Labeling oriented links according to the signed crossing conditions

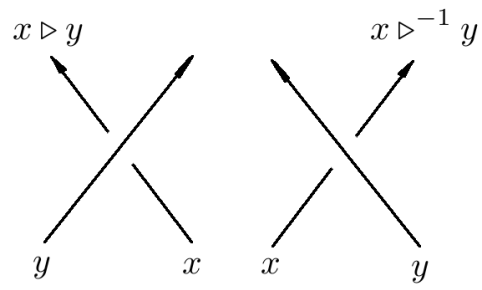

defines homomorphisms from the fundamental quandle of the link $L$ into $X$; the integral quandle counting invariant $\Phi_{X}^{\mathbb{Z}}(L)$ is then an invariant of oriented links.

\section{Kei algebras and modules}

Let $X$ be a finite kei. We would like to define an associative algebra on $X$ generated by "beads" such that secondary labelings of $X$-labeled link diagrams by beads are preserved by Reidemeister moves. Specifically, at a crossing in a link diagram with arcs labeled $x, y$ and $x \triangleright y$, we define the following relationship between the beads $a$, $b$, and $c$ :

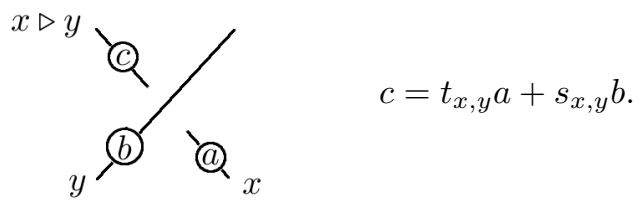


The kei algebra of $X, \mathbb{Z}_{K}[X]$, is the quotient of the polynomial algebra $\mathbb{Z}\left[t_{x, y}, s_{x, y}\right]$ by the ideal $I$ required to obtain invariance under unoriented Reidemeister moves.

First, we note that the bead relationship above also requires that

$$
a=t_{x \triangleright y, y} c+s_{x \triangleright y, y} b ;
$$

together these imply

$$
a=t_{x, y} t_{x \triangleright y, y} a+\left(t_{x, y} s_{x \triangleright y, y}+s_{x, y}\right) b,
$$

which yields

$$
t_{x, y} t_{x \triangleright y, y}=1 \quad \text { and } \quad t_{x, y} s_{x \triangleright y, y}+s_{x, y}=0 .
$$

From the Reidemeister I move, we must have $t_{x, x}+s_{x, x}=1$ :

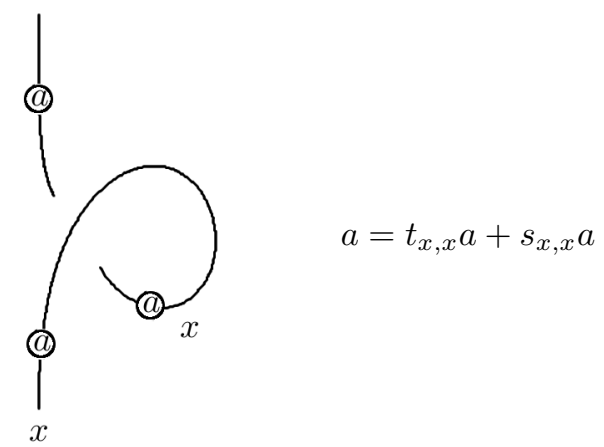

The Reidemeister II move yields conditions equivalent to equation (1):

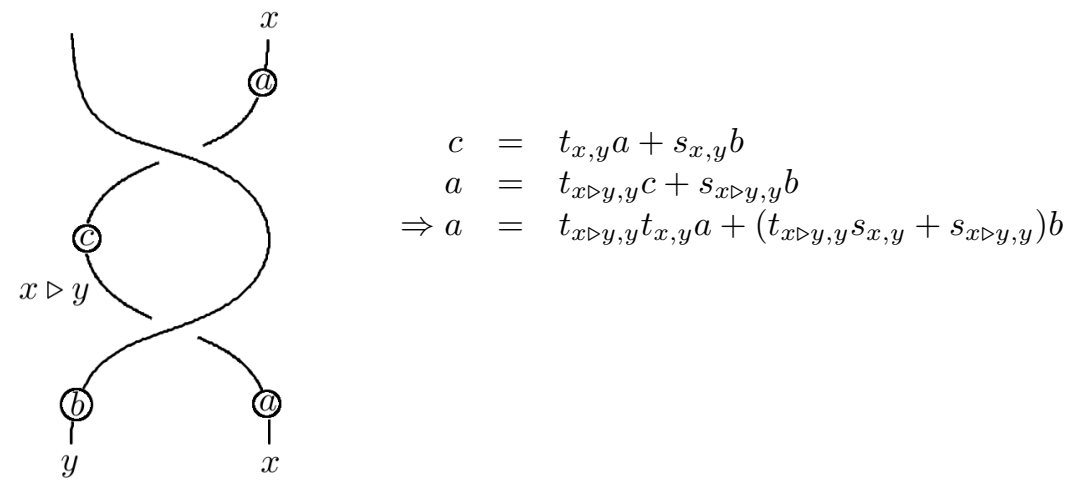


The Reidemeister III move yields the defining equations for the original rack algebra $\mathbb{Z}[X]$ from $[\mathbf{1}]$, comparing coefficients on bead $e$ :

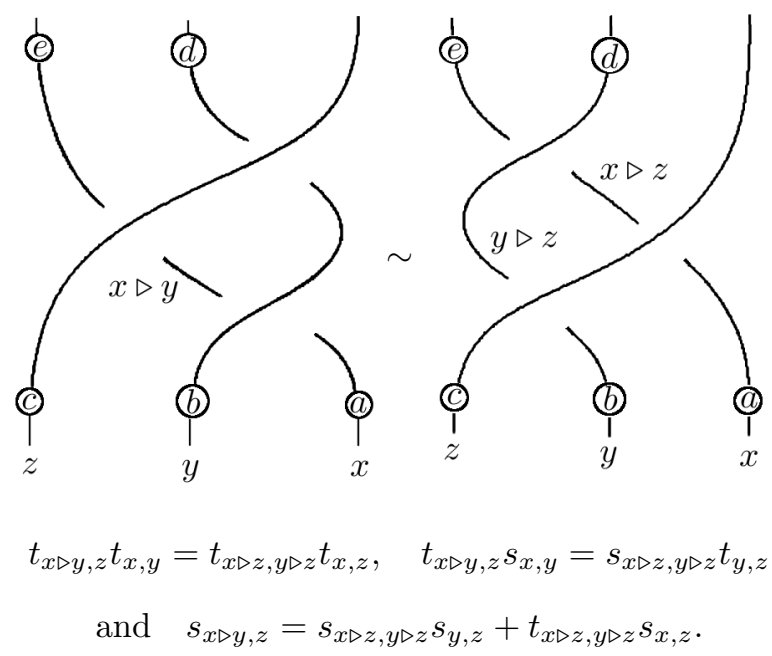

Definition 3.1. Let $X$ be a finite kei. The kei algebra $\mathbb{Z}_{K}[X]$ of $X$ is the quotient of the polynomial algebra $\mathbb{Z}\left[t_{x, y}, s_{x, y}\right]$ for all $x, y \in X$ by the ideal $I$ generated by all elements of the forms

$$
\begin{array}{ll}
\text { - } t_{x, y} s_{x \triangleright y, y}+s_{x, y} & \bullet t_{x, y} t_{x \triangleright y, y}-1 \\
\text { - } t_{x, x}+s_{x, x}-1 & \bullet t_{x \triangleright y, z} t_{x, y}-t_{x \triangleright z, y \triangleright z} t_{x, z} \\
\text { - } t_{x \triangleright y, z} s_{x, y}-s_{x \triangleright z, y \triangleright z} t_{y, z} & \bullet s_{x \triangleright y, z}-s_{x \triangleright z, y \triangleright z} s_{y, z}-t_{x \triangleright z, y \triangleright z} s_{x, z}
\end{array}
$$

for all $x, y, z \in X$. A $\mathbb{Z}_{K}[X]$-module or just an $X$-module is a representation of $\mathbb{Z}_{K}[X]$, i.e. an abelian group $A$ with a family of automorphisms $t_{x, y}: A \rightarrow A$ and endomorphisms $s_{x, y}: A \rightarrow A$ such that each of the bulleted maps listed above are zero.

We can now define the kei algebra of a finite kei $X$.

Example 3.2. Let $X$ be a kei. Any ring $R$ becomes a $\mathbb{Z}_{K}[X]$-module by choosing invertible elements $t_{x, y}$ and elements $s_{x, y}$ for $x, y \in X$ satisfying the conditions (1), (2), and (3). In particular, if $X=\left\{x_{1}, x_{2}, \ldots, x_{n}\right\}$ is a finite kei, we can specify a $\mathbb{Z}_{K}[X]$-module structure on $R$ with an $n \times 2 n$ block matrix

$$
M_{R}=\left[\begin{array}{cccc|cccc}
t_{1,1} & t_{1,2} & \ldots & t_{1, n} & s_{1,1} & s_{1,2} & \ldots & s_{1, n} \\
t_{2,1} & t_{2,2} & \ldots & t_{2, n} & s_{2,1} & s_{2,2} & \ldots & s_{2, n} \\
\vdots & \vdots & \ddots & \vdots & \vdots & \vdots & \ddots & \vdots \\
t_{n, 1} & t_{n, 2} & \ldots & t_{n, n} & s_{n, 1} & s_{n, 2} & \ldots & s_{n, n}
\end{array}\right]
$$

such that the entries satisfy the conditions (1), (2), and (3) above.

Remark 3.3. The quandle algebra defined in [1] is the quotient of the polynomial algebra $\mathbb{Z}\left[t_{x, y}^{ \pm 1}, s_{x, y}\right]$ by the ideal generated by the relations coming from the Reidemeister I and III moves, i.e.,

- $t_{x, x}+s_{x, x}-1$ 
- $t_{x \triangleright y, z} t_{x, y}-t_{x \triangleright z, y \triangleright z} t_{x, z}$

- $t_{x \triangleright y, z} s_{x, y}-s_{x \triangleright z, y \triangleright z} t_{y, z}$,

- $s_{x \triangleright y, z}-s_{x \triangleright z, y \triangleright z} s_{y, z}-t_{x \triangleright z, y \triangleright z} s_{x, z}$,

with the type II move condition handled by the bead labeling rule below.

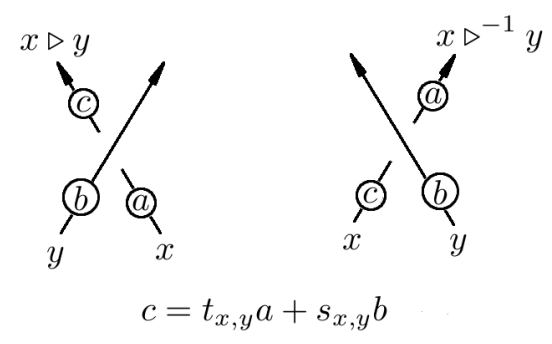

The kei algebra $\mathbb{Z}_{K}[X]$ is a quotient of the quandle algebra by the additional relations

$$
t_{x, y} s_{x \triangleright y, y}+s_{x, y} \quad \text { and } \quad 1-t_{x, y} t_{x \triangleright y, y} .
$$

Example 3.4. For a specific instance of the type of kei module defined in example 3.2, let $X$ be the 3 -element Takasaki kei with kei matrix

$$
M_{X}=\left[\begin{array}{lll}
1 & 3 & 2 \\
3 & 2 & 1 \\
2 & 1 & 3
\end{array}\right]
$$

and let the ring $R=\mathbb{Z}_{5}$. Our python computations indicate that there are $48 \mathbb{Z}_{K}[X]$ module structures on $R$, including for instance

$$
M_{R}=\left[\begin{array}{lll|lll}
4 & 1 & 3 & 2 & 4 & 1 \\
3 & 4 & 2 & 3 & 2 & 3 \\
2 & 1 & 4 & 4 & 1 & 2
\end{array}\right] .
$$

Remark 3.5. For a given kei $X$, the set of $\mathbb{Z}_{K}[X]$-modules over a given ring $R$ is a subset of the set of $\mathbb{Z}[X]$-modules, and can be a proper subset depending on $R$, since a $\mathbb{Z}[X]$-module satisfies the conditions in equations (1) and (3) but not necessarily those of equation (2). For instance, our python computations reveal a total of 32 $\mathbb{Z}[X]$-modules on the kei $X$ and ring $R$ in example 3.4 which are not $\mathbb{Z}_{K}[X]$-modules, including for instance

$$
M_{R}=\left[\begin{array}{lll|lll}
2 & 1 & 2 & 4 & 2 & 3 \\
1 & 2 & 2 & 2 & 4 & 3 \\
4 & 4 & 2 & 4 & 4 & 4
\end{array}\right] .
$$

The invariants defined in the next section associated with such modules are invariants of oriented links but not invariants of unoriented links.

Example 3.6. Another important example of a $\mathbb{Z}_{K}[X]$ module is the fundamental $\mathbb{Z}_{K}[X]$-module of an $X$-labeled link. Let $L$ be an unoriented link with a labeling $f: F K(L) \rightarrow X$ by a kei $X$. On each arc of $L$, we place a bead; the set of crossing relations then determines a presentation for a $\mathbb{Z}_{K}[X]$-module, denoted $\mathbb{Z}_{f}[X]$, which 
we can represent concretely with a coefficient matrix of the resulting homogeneous system of linear equations. For instance, let $X$ be the kei with matrix

$$
M_{X}=\left[\begin{array}{lll}
1 & 1 & 2 \\
2 & 2 & 1 \\
3 & 3 & 3
\end{array}\right] ;
$$

then the $(4,2)$-torus link with the $X$-labeling below has fundamental $\mathbb{Z}_{k}[X]$-module presented by the matrix $M_{\mathbb{Z}_{f}[X]}$ :

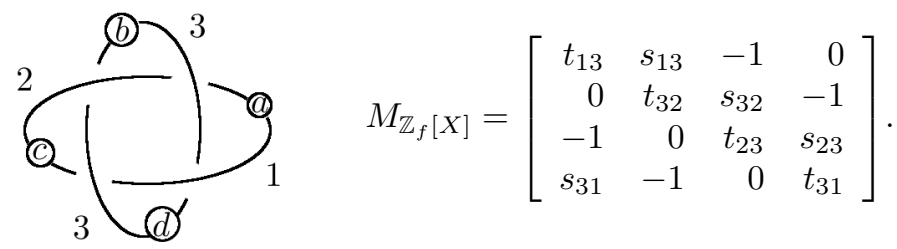

\section{Kei module enhancements of the counting invariant}

We can now define invariants of unoriented knots and links using kei modules. The idea is to use the set of homomorphisms $g: \mathbb{Z}_{f}[X] \rightarrow R$ from the fundamental kei module of an $X$-labeled diagram $L$ to the kei module $R$ as a signature for each kei homomorphism $f: F K(L) \rightarrow X$.

Definition 4.1. Let $L$ be an unoriented knot or link, $X$ a finite kei, and $R$ a finite $\mathbb{Z}_{K}[X]$-module. The kei module enhanced multiset invariant of $L$ associated to $X$ and $R$ is the multiset of cardinalities of the sets of $\mathbb{Z}_{k}[X]$-module homomorphisms, i.e.,

$$
\Phi_{X, R}^{K, M}(L)=\left\{\left|\operatorname{Hom}_{\mathbb{Z}_{K}[X]}\left(\mathbb{Z}_{f}[X], R\right)\right|: f \in \operatorname{Hom}(F K(L), X)\right\} .
$$

Taking the generating function of this multiset gives us a polynomial-form invariant for easy comparison: the kei module enhanced invariant of $L$ with respect to $X$ and $R$ is

$$
\Phi_{X, R}^{K}(L)=\sum_{f \in \operatorname{Hom}(F K(L), X)} u^{\left|\operatorname{Hom}_{\mathbb{Z}_{K}[X]}\left(\mathbb{Z}_{f}[X], R\right)\right|} .
$$

By construction, we have the following:

Theorem 4.2. If $L$ and $L^{\prime}$ are ambient isotopic unoriented links, $X$ is a finite kei, and $R$ is a $\mathbb{Z}_{K}[X]$-module, then $\Phi_{X, R}^{K, M}(L)=\Phi_{X, R}^{K, M}\left(L^{\prime}\right)$ and $\Phi_{X, R}^{K}(L)=\Phi_{X, R}^{K}\left(L^{\prime}\right)$.

Remark 4.3. If $R$ is not finite, we can replace the cardinality $\left|\operatorname{Hom}_{\mathbb{Z}_{K}[X]}\left(\mathbb{Z}_{f}[X], R\right)\right|$ with the rank of the $\mathbb{Z}_{K}[X]$-module $\operatorname{Hom}_{\mathbb{Z}_{K}[X]}\left(\mathbb{Z}_{f}[X], R\right)$.

To compute $\Phi_{X, R}^{K}$, for each kei labeling $f: F K(L) \rightarrow X$ of $L$ by $X$, we first obtain the matrix for $\mathbb{Z}_{f}[X]$, replace each $t_{x, y}$ and $s_{x, y}$ with its value in $R$, and solve the resulting system of equations to obtain the contributions to $\Phi_{X, R}^{K}$ for $f$.

Example 4.4. Let $L$ be the figure eight knot $4_{1}$ and let $X$ and $R$ be the kei and kei module on $\mathbb{Z}_{5}$ from example 3.4. The set of $X$-labelings of $L$ includes only constant 
labelings, i.e. every arc is labeled with a 1,2 , or 3 . For example, the constant labeling with every arc labeled 1 yields the listed $\mathbb{Z}_{f}[X]$-presentation matrix:

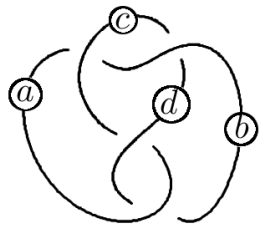

$$
M_{\mathbb{Z}_{f}[X]}=\left[\begin{array}{rrrr}
t_{11} & -1 & s_{11} & 0 \\
0 & s_{11} & t_{11} & -1 \\
-1 & 0 & t_{11} & s_{11} \\
s_{11} & t_{11} & 0 & -1
\end{array}\right]
$$

Replacing the $t_{x y}$ and $s_{x, y}$ with their values in $R$ and row-reducing over $\mathbb{Z}_{5}$, we obtain

$$
\left[\begin{array}{llll}
4 & 4 & 2 & 0 \\
0 & 2 & 4 & 4 \\
4 & 0 & 4 & 2 \\
2 & 4 & 0 & 4
\end{array}\right] \longrightarrow\left[\begin{array}{llll}
1 & 1 & 3 & 0 \\
0 & 1 & 2 & 2 \\
0 & 0 & 0 & 0 \\
0 & 0 & 0 & 0
\end{array}\right]
$$

and this $X$-labeling contributes a $u^{25}$ to the invariant $\Phi_{X, R}^{k}\left(4_{1}\right)$. Summing these contributions over the complete set of $X$-labelings gives us $\Phi_{X, R}^{K}\left(4_{1}\right)=3 u^{25}$. Comparing this to the unknot, which has $\Phi_{X, R}^{k}($ Unknot $)=3 u^{5}$, we see that $\Phi_{X, R}^{K}$ distinguishes the unoriented figure eight from the unoriented unknot despite the two having equal kei counting invariant values. In particular, since $\Phi_{X}^{\mathbb{Z}}(k)$ is obtained from $\Phi_{X, R}^{K}$ by evaluating at $u=1, \Phi_{X, R}^{K}$ is a strictly stronger invariant than $\Phi_{X}^{\mathbb{Z}}(k)$.

Example 4.5. Our python computations ${ }^{1}$ yield the listed values for $\Phi_{X, R}^{K}$ with $X$ the 3 -element Takasaki kei and the randomly selected $\mathbb{Z}_{K}[X]$-module over $\mathbb{Z}_{7}$ below for the prime knots with up to eight crossings and prime links with up to seven crossings as listed in the knot atlas [2]:

$$
M_{R}=\left[\begin{array}{lll|lll}
6 & 3 & 5 & 2 & 5 & 3 \\
5 & 6 & 3 & 3 & 2 & 5 \\
3 & 5 & 6 & 5 & 3 & 2
\end{array}\right]
$$

\begin{tabular}{r|l}
$\Phi_{X, M}^{K}(L)$ & $L$ \\
\hline $3 u^{7}$ & unknot, $4_{1}, 5_{1}, 6_{2}, 6_{3}, 7_{2}, 7_{3}, 7_{5}, 7_{6}, 8_{1}, 8_{2}, 8_{3}, 8_{4}, 8_{6}, 8_{7}, 8_{8}, 8_{9}, 8_{12}, 8_{13}$, \\
& $8_{14}, 8_{17}, L 2 a 1, L 4 a 1, L 6 a 2, L 6 a 4, L 6 n 1, L 7 a 2, L 7 a 3, L 7 a 4, L 7 a 7$, \\
& $L 7 n 1, L 7 n 2$ \\
$3 u^{7}+6 u^{49}$ & $3_{1}, 6_{1}, 7_{4}, 8_{10}, 8_{11}, 8_{15}, 8_{19}, 8_{20}, 8_{21}, L 6 a 1, L 6 a 3, L 6 a 5, L 7 a 1, L 7 a 5$ \\
$3 u^{7}+24 u^{49}$ & $8_{18}$ \\
$3 u^{49}$ & $5_{2}, 7_{1}, 8_{16}, L 7 a 6$ \\
$9 u^{49}$ & $7_{7}, 8_{5}$
\end{tabular}

Remark 4.6. As with most enhancements of quandle-related counting invariants, $\Phi_{X, M}^{K}$ is well-defined for unoriented virtual links as well as classical links.

\footnotetext{
${ }^{1}$ Code available at http://www.esotericka.org.
} 
In our final example, we use a quandle module which is not a kei module to detect the non-invertibility of a virtual knot.

Example 4.7. Let $X$ be the kei from example 3.4 and $M$ the quandle module from remark 3.5. Since $M$ is not a kei module, $\Phi_{X}^{M}$ is an invariant of oriented knots and links, but not unoriented knots and links. Thus, we can potentially use $\Phi_{X}^{M}$ to compare the two orientations of a non-invertible knot. In particular, consider the virtual knot numbered 4.97 in the Knot Atlas [2]; it is the closure of the virtual braid below. Let us denote 4.97 with the upward orientation by $4.97 \uparrow$ and 4.97 with the downward orientation as $4.97_{\downarrow}$. The only labelings of 4.97 by $X$ are constant labelings, of which there are three for both orientations, the unenhanced integral kei counting invariant $\Phi_{X}^{\mathbb{Z}}\left(4.97_{\uparrow}\right)=3=\Phi_{X}^{\mathbb{Z}}\left(4.97_{\downarrow}\right)$, and $\Phi_{X}^{\mathbb{Z}}$ does not distinguish $4.97_{\uparrow}$ from $4.97_{\downarrow}$. However, the constant labeling with every arc labeled with a $1 \in X$ yields the listed fundamental kei module presentation matrices. Replacing $t_{1,1}$ and $s_{1,1}$ with their values from $M$ yields the listed matrices, which we row-reduce over $\mathbb{Z}_{5}$ to obtain the cardinalities of the solution spaces which form the signature of the constant labeling by the element $1 \in X$.

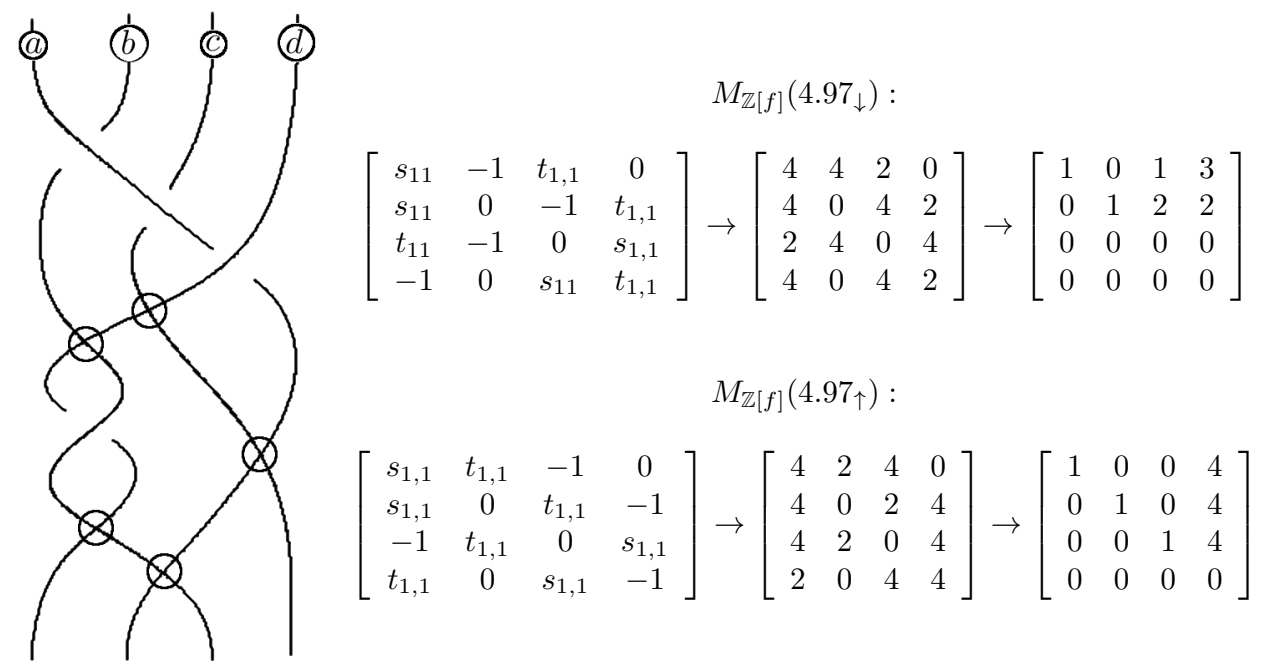

Since $t_{1,1}=t_{2,2}=t_{3,3}=2$ and $s_{1,1}=s_{2,2}=s_{3,3}=4$, we get the same signatures for all three labelings for each knot, respectively $u^{25}$ and $u^{5}$, and thus we have

$$
\Phi_{X}^{M}\left(4.97^{\downarrow}\right)=3 u^{25} \neq 3 u^{5}=\Phi_{X}^{M}\left(4.97_{\uparrow}\right)
$$

and for non-kei module quandle modules $M$ over a finite kei, $X$, the quandle module enhanced counting invariant $\Phi_{X}^{M}$ is capable of detecting invertibility of virtual (and hence classical) knots.

\section{Questions}

In this section we collect a few open questions for future research.

In our computations we have only considered the simplest type of $\mathbb{Z}_{K}[X]$ modules, namely $\mathbb{Z}_{k}[X]$-module structures on $\mathbb{Z}_{n}$ with the action of $t_{x, y}$ and $s_{x, y}$ given by 
multiplication by fixed elements of $\mathbb{Z}_{K}[X]$. Expanding to other abelian groups with other automorphisms $t_{x, y}: X \rightarrow X$ and endomorphisms $s_{x, y}: X \rightarrow X$ should give interesting results. We are particularly interested in the case of non-commuting $t_{x, y}$ and $s_{x, y}$ values.

We have generalized the rack module bead counting invariant from [8], but several other oriented link invariants using the quandle algebra were defined in [3]; these invariants should have generalizations to the unoriented case using the kei algebra.

\section{References}

[1] N. Andruskiewitsch and M. Graña. From racks to pointed Hopf algebras. Adv. Math. 178 (2003) 177-243.

[2] D. Bar-Natan (ed.). The Knot Atlas. http://katlas.math.toronto.edu/ wiki/Main_Page

[3] J.S. Carter, M. Elhamdadi, M. Graña and M. Saito. Cocycle knot invariants from quandle modules and generalized quandle homology. Osaka J. Math. 42 (2005) 499-541.

[4] J.S. Carter and M. Saito. Set-Theoretic Yang-Baxter Solutions via Fox Calculus. J. Knot Theory Ramifications 15 (2006) 949-956.

[5] R. Fenn and C. Rourke. Racks and links in codimension two. J. Knot Theory Ramifications 1 (1992) 343-406.

[6] B. Ho and S. Nelson. Matrices and finite quandles. Homology Homotopy Appl. 7 (2005) 197-208.

[7] D. Joyce. A classifying invariant of knots, the knot quandle. J. Pure Appl. Algebra 23 (1982) 37-65.

[8] A. Haas, G. Heckel, S. Nelson, J. Yuen and Q. Zhang Rack Module Enhancements of Counting Invariants. To appear in Osaka J. Math., arXiv:1008.0114.

[9] S.V. Matveev. Distributive groupoids in knot theory. Math. USSR, Sb. 47 (1984) 73-83.

[10] M. Takasaki. Abstractions of symmetric functions. Tohoku Math. J. 49 (1943) 143-207.

Michael Grier mhg02007@mymail.pomona.edu

Sam Nelson knots@esotericka.org

Department of Mathematical Sciences, Claremont McKenna College, 850 Columbia Ave., Claremont, CA 91711, USA 